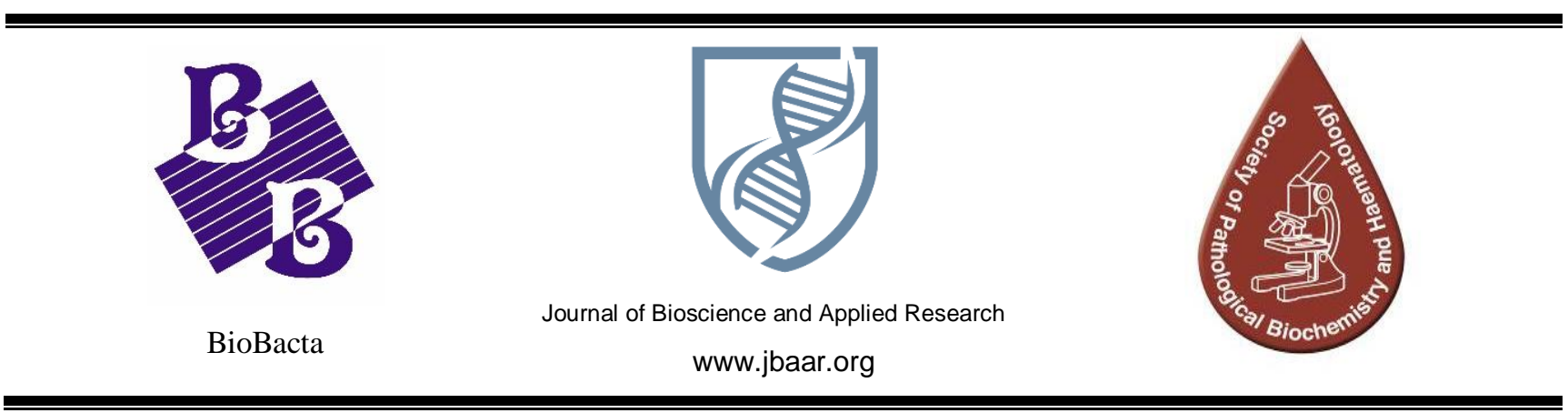

\title{
Kinetic and conductivity study to oxidation drug ketone, using iodoform in basic medium
}

\author{
Layla A.Al-Juber*, Gazwan H. Al-Somaidaie**, Ayad S.Hamed ${ }^{* *}$ \\ Shemaa H.Abdullah** \\ *Physical Chemistry, ** Organic Chemistry \\ Department of Chemistry, College of Education for Pure Sciences, University of Tikrit, Iraq \\ Received: March 10, 2018. Accepted: June 5, 2018. Published: June 14, 2018
}

DOI: 10.21608/JBAAR.2018.130271

\begin{abstract}
Iodoform oxidation for drug ketone (spusforonion) at room temperature by using the conductivity study and spectrophotometric in basic medium. The conductivity study showed that molar conductance values were decreased with increasing of time at $\left(2 \times 10^{-4}-4 \times 10^{-4}\right)$ concentration, while at $\left(5 \times 10^{-4}-6 \times 10^{-4}\right)$ the value of molar conductance was increased, due to the reaction in presence of basic medium and apply the Helmy's theory of oxidation solutions was applied in ethanol for the initial infinity Molaric conductivity $\Lambda_{\circ}$ at $25^{0}{ }_{\text {was }}^{\circ}$ found the linear relationship between the $\ln \Lambda$ and $C^{1 / 2}$ for the weak electrodes. The second study was spectrophotometric in a basic medium which showed that the reaction is related to the first order for the oxidation drug concentration by the iodoform.
\end{abstract}

Key Words: Iodoform oxidation, drug ketone, spusforonion, conductivity

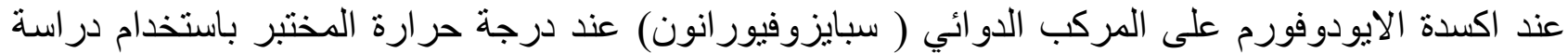

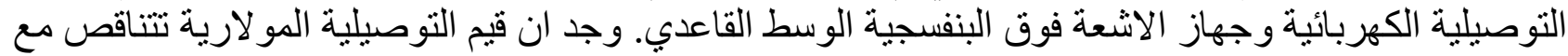
زيادة الزمن عند التراكيز(4)

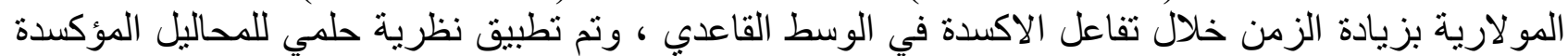

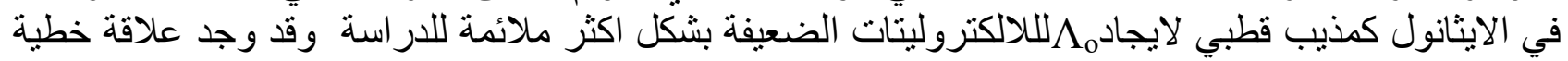

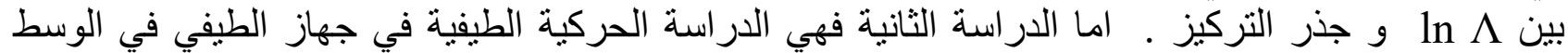

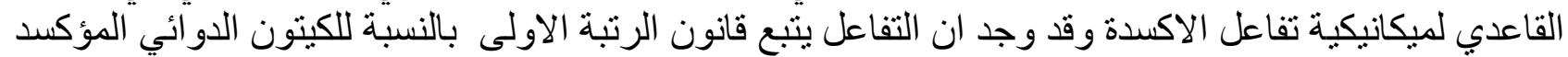




\section{Introduction}

This study was divided into two parts. The first part included the study of the chemical ketone oxidation using the electrolysis and the second part included the kinetic study of the oxidation of the ketone drug using the iodoform. Oxidation is a term that refers to the degree of oxidation for an atom of a chemical compound[1]. The conception of oxidation state the electrical charge which is gained by another atom that sharing an ionic bond when connecting with others in $100 \%$. The oxidation state is represented in true numbers, could be negative, positive signal, or even zero[2]. in some cases, the oxidation state could be a fraction value such as 3/8 for Iron in $\left(\mathrm{Fe}_{3} \mathrm{O}_{4}\right)$. The higher degree of oxidation is $(+8)$ in $\mathrm{XeO}_{4}, \mathrm{ReO}_{4}$, and $\mathrm{OsO}_{4}$, while the lower ones are (-4) which were found in some of the carbon group elements[3]. The increase in oxidation state for an atom during the chemical reaction is called "oxidation", Oxidation is a key reaction for different organic synthesis. While the decreasing called "reduction"[3]. These reactions involve the transformation of electrons, the accepting of electrons is regarded as a reduction while the loss of electron is oxidation[4]. Chemical Kinetics of the substance is exactly how reactant set changed over into things furthermore of all physical and compound strategies which happen in midst obviously of reaction part of reaction gives a clear photograph of a sanctioned complex[5]. It is said that "Framework is to Science as semantic use is to lingo". Examination of vitality is truly isolated into two areas. IUPAC defines the oxidation state as follows[6] :

1- The oxidation state for the monoatomic ion is equal to its charge.

2- Oxidation state to free element (unconnected)equal zero.

3- Oxidation state for hydrogen equal $(+1)$, oxygen (-2), when they exist in most of the compounds in special cases the oxidation of hydrogen is (-1) as inactive metals hydrides, like $\mathrm{LiH}$, and the oxidation state in peroxides is (-1) like $\mathrm{H}_{2} \mathrm{O}_{2}$.

4- The total combination of the oxidation state of atoms in isoelectric molecules must be zero while in atoms which form ions must be equal to its charge, for example, the oxidation state of sulpher in $\mathrm{H} 2 \mathrm{~S}$, S8(primary sulpher), $\mathrm{SO}_{2}, \mathrm{SO}_{3}$, and $\mathrm{H}_{2} \mathrm{SO}_{4}$ is equal: $-2,0,+4$, and +6 respectively. The oxidation state for the atom would be higher, in case of increasing oxidation, and would be lower in case of increasing reduction. iodoform is an important and useful tool in the hands of chemists both for preparative and analytical purposes[7]. The utility of iodoform as an oxidant in the kinetic study may be attributed to the vital role of it in various iodoform synthesis also in various media such as basic or even in organic solvents[7]. The present investigation reports the oxidation of the drug ketone by iodoform under first-order reaction conditions in a basic medium.

The second part involves the Conductivity study, which is the equivalent conductance $(\Lambda)$ of electrolyte is defined as the conductivity $1 \mathrm{~cm} 3$ volume, contains one gram equivalence[8]. the Equivalent conductance is represented by[8] :

$$
\Lambda=L x V
$$

$\Lambda=\frac{L \cdot 1000}{C}$

Where $\mathrm{L}=$ Specific conductivity

$\mathrm{V}=$ Volume of solution in cc. containing one gram equivalent of the electrolyte.

Two effects that are acting to prevent the mobility of the ions are :1- Asymmetric effect, 2electrophoretic effect. Onsager equation (Kohlrausch's Law) used those effects as follows[9] :

dilution

$\Lambda=\Lambda_{0}-\left(\mathrm{A}+\mathrm{B} \Lambda_{\mathrm{o}}\right) \mathrm{C}^{1 / 2}$

Where $\Lambda_{0}=$ molar conductance at infinite

Kinetics provides useful information about the mechanism and rate of chemical reaction, which helps to run a chemical reaction successfully by a way of selecting optimum conditions to get the maximum yield[10]. The kinetic study also helps us to study the factors which influence the rate of reaction like temperature, pressure, substrate concentration, oxidant concentration, the composition of the reaction mixture, and catalyst [11]. The reaction kinetics plays a very important role in the investigation of the reaction mechanism. Oxidation of organic compound carried out by oxidizing agent like iodoform[12-14].iodoform used to carry out the kinetics and mechanism of various organic compounds. The first-order reaction is dependent on concentration change with time[15].

$\ln \left(\frac{a}{a-x}\right)=k \cdot t$ 
The (a) is initial concentration and (a-x) is reaction materials concentration.

This study aims to show the change in kinetic of drug ketone with iodoform tracing with a spectrophotometer, electro study and find the rate constant, finding a degree of dissociation for the reaction formed between drug ketone and iodoform, and finding dissociation " constant Kc".

The mechanism Iodoform synthesis as in the following diagrams[7]

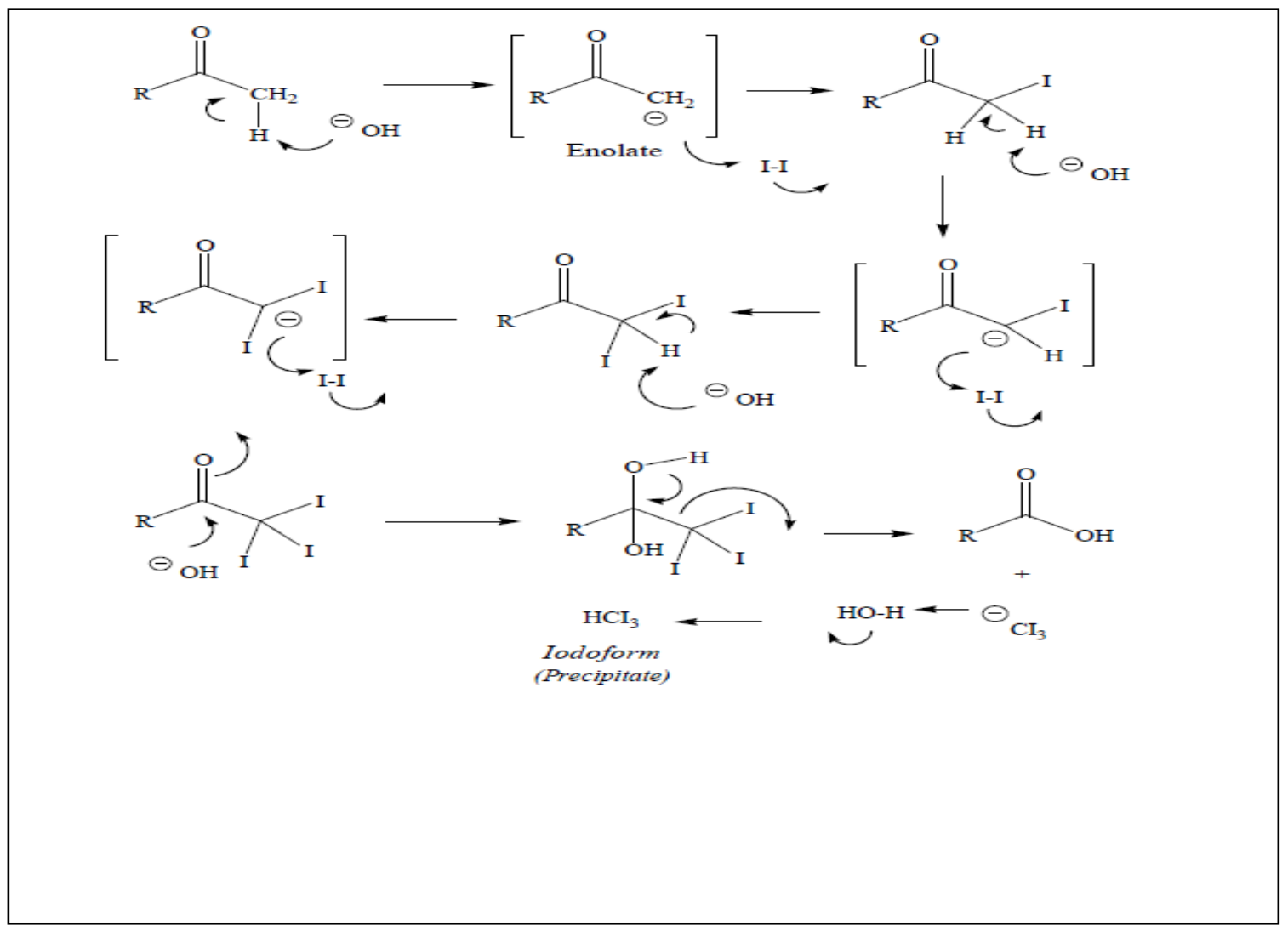

\section{MATERIALS AND METHODS}

All chemicals were reagent grade or better and used without being further purified.

ketone drug :5-Acetyl Spiro [benzofuran $2(3 \mathrm{H})$,Iprime-Cyclopropan]-3-one $\left(\mathrm{C}_{12} \mathrm{H}_{18} \mathrm{O}_{3}\right)$ the concentration is $(0.0001 \mathrm{M})$

sodium hydroxide $(0.002 \mathrm{M})$, Iodine $(0.0001 \mathrm{M})$, potassium iodide $(0.0001 \mathrm{M})$, all this is solvent ethanol absolute .

the apparatus used is: Uv/vis spectrometers T90+ two-beam and spectrophotometers one beam, conductivity is type Lab 720 INLAB . procedure[12]:

1- Oxidation prepared (reagent) $(0.001 \mathrm{M})$ of $\mathrm{I} 2, \quad(0.00025 \mathrm{gm})$ was dissolved in a beaker canting $(5 \mathrm{ml})$ of ethanol, in another canting $(5 \mathrm{ml})$ of ethanol $(0,0001 \mathrm{M})$ of $\mathrm{KI}(0.0016 \mathrm{gm})$ was also dissolved, after completion of dissolving the two beakers, they were mixed to have new reagent with wavelength $=363 \mathrm{~nm}$.

2- Preparation of drug ketone

$(0.001 \mathrm{M})$ of the ketone $(0.0021 \mathrm{gm})$ was dissolved in $(10 \mathrm{ml})$ of ethanol in a beaker with wavelength $=325 \mathrm{~nm}$.

3- $\mathrm{NaOH}$ preparation 
$(0.002 \mathrm{M})$ of the ketone $(0.0008 \mathrm{gm})$ was dissolved in $(10 \mathrm{ml})$ of ethanol in a beaker

4- preparation of Mixture

The solution of ketone and base were mixed for five minutes, after that the reagent containing $(\mathrm{KI}+\mathrm{I} 2)$ was added in a volumetric flask with $10 \mathrm{ml}$ capacity, the volume was completed to $10 \mathrm{ml}$ by adding absolute ethanol using a range of ketone concentration, wavelength for the mixture is $498 \mathrm{~nm}$.

Table(1): show the volumes using for (drug ketone, oxidation, and base volume )

\begin{tabular}{|c|c|c|c|c|c|}
\hline $\begin{array}{c}\text { Ethanol } \\
\text { volume }\end{array}$ & $\begin{array}{c}\text { volume of } \\
\text { Oxidation }\end{array}$ & $\begin{array}{c}\text { Volume of } \\
\mathrm{NaOH}\end{array}$ & $\begin{array}{c}\text { The } \\
\text { volume of } \\
\text { drug } \\
\text { ketone }\end{array}$ & Volume & Concentration \\
\hline $7 \mathrm{ml}$ & $1 \mathrm{ml}$ & $1 \mathrm{ml}$ & $1 \mathrm{ml}$ & $1 \mathrm{ml}$ & $2 \times 10^{-4}$ \\
\hline $6 \mathrm{ml}$ & $1.5 \mathrm{ml}$ & $1.5 \mathrm{ml}$ & $1 \mathrm{ml}$ & $1.5 \mathrm{ml}$ & $3 \times 10^{-4}$ \\
\hline $5 \mathrm{ml}$ & $2 \mathrm{ml}$ & $2 \mathrm{ml}$ & $1 \mathrm{ml}$ & $2 \mathrm{ml}$ & $4 \times 10^{-4}$ \\
\hline $4 \mathrm{ml}$ & $2.5 \mathrm{ml}$ & $2.5 \mathrm{ml}$ & $1 \mathrm{ml}$ & $2.5 \mathrm{ml}$ & $5 \times 10^{-4}$ \\
\hline $3 \mathrm{ml}$ & $3 \mathrm{ml}$ & $3 \mathrm{ml}$ & $1 \mathrm{ml}$ & $3 \mathrm{ml}$ & $6 \times 10^{-4}$ \\
\hline $2 \mathrm{ml}$ & $3.5 \mathrm{ml}$ & $3.5 \mathrm{ml}$ & $1 \mathrm{ml}$ & $3.5 \mathrm{ml}$ & $7 \times 10^{-4}$ \\
\hline $1 \mathrm{ml}$ & $4 \mathrm{ml}$ & $4 \mathrm{ml}$ & $1 \mathrm{ml}$ & $4 \mathrm{ml}$ & $8 \times 10^{-4}$ \\
\hline
\end{tabular}

\section{RESULTS AND DISCUSSION}

\section{1- Results of conductivity experiments}

The reaction kinetics was studied using conductivity meter, the following results were obtained via the tables (2-6) shown below the molar conductance of drug ketone had been calculated with oxidation factor, according to general law :

$\Lambda=\frac{1000 L}{C}$

Conductance at infinite dilution, according to Kohlrausch's Law, was also calculated, through plotting the relationship between equivalent conductance versus the square root of concentration that shown, the equivalent conductance for the synthesized compound with oxidized factor, is weak : $\wedge=\wedge \circ-(A+B . \wedge \circ), \sqrt{c}$

The study stated that the conductivity for these compounds, is weak and few, according to the values of dissociation reaction, which was calculated according to the following equation :

$\propto=\frac{\Lambda}{\Lambda_{0}}$

It is stated in table (7) and figure (6), this is due to the occupation of electronic pair for drug ketone during the addition because of increasing the number of collisions per time. for this values of conductivity decreased, indicating that the synthesized compound is a weak electrolyte. 
Table (1)

state conductivity values and rate constant for the effect of oxidation factors at [iodoforme] $=2 \times 10^{-4}$

\begin{tabular}{|c|c|c|c|}
\hline Time(min) & $\mathrm{L} \mu \mathrm{S} / \mathrm{cm}$ & $\begin{array}{c}\Lambda \\
\text { s.eq }^{-1} . \mathrm{cm}^{2}\end{array}$ & $\mathrm{~K}\left(\mathrm{~min}^{-1}\right)$ \\
\hline 0 & 31.0 & 15.5 & \multirow{16}{*}{$5.12 \times 10^{-3}$} \\
\hline 2 & 30.6 & 15.3 & \\
\hline 4 & 30.4 & 15.2 & \\
\hline 6 & 30.2 & 15.1 & \\
\hline 8 & 30.0 & 15 & \\
\hline 10 & 29.9 & 14.95 & \\
\hline 12 & 29.8 & 14.9 & \\
\hline 14 & 29.5 & 14.75 & \\
\hline 16 & 27.9 & 13.95 & \\
\hline 18 & 27.8 & 13.9 & \\
\hline 20 & 27.7 & 13.85 & \\
\hline 22 & 27.6 & 13.8 & \\
\hline 24 & 27.5 & 13.75 & \\
\hline 26 & 27.4 & 13.7 & \\
\hline 28 & 27.3 & 13.56 & \\
\hline 30 & 27.2 & 13.6 & \\
\hline
\end{tabular}

Table (2)

state conductivity values and rate constant for the effect of oxidation factors at [iodoforme] $=3 \times 10^{-4}$

\begin{tabular}{|c|c|c|c|}
\hline Time(min) & $\mathrm{L} \mu \mathrm{S} / \mathrm{cm}$ & $\begin{array}{c}\Lambda \\
\text { s.eq }^{-1} \cdot \mathrm{cm}^{2}\end{array}$ & $\mathrm{~K}\left(\min ^{-1}\right)$ \\
\hline 0 & 16.25 & 5.41 & \multirow{16}{*}{$2.78 \times 10^{-3}$} \\
\hline 2 & 16.12 & 5.37 & \\
\hline 4 & 15.83 & 5.27 & \\
\hline 6 & 15.51 & 5.21 & \\
\hline 8 & 15.63 & 5.17 & \\
\hline 10 & 15.23 & 5.09 & \\
\hline 12 & 15.28 & 5.07 & \\
\hline 14 & 14.82 & 4.94 & \\
\hline 16 & 14.77 & 4.92 & \\
\hline 18 & 14.77 & 4.90 & \\
\hline 20 & 14.52 & 4.84 & \\
\hline 22 & 14.41 & 4.80 & \\
\hline 24 & 14.10 & 4.76 & \\
\hline 26 & 13.87 & 4.62 & \\
\hline 28 & 13.84 & 4.61 & \\
\hline 30 & 13.78 & 4.59 & \\
\hline
\end{tabular}


Table (3)

state conductivity values and rate constant for the effect of oxidation factors at [iodoforme] $=4 \times 10^{-4}$

\begin{tabular}{|c|c|c|c|}
\hline Time(min) & $\mathrm{L} \mu \mathrm{S} / \mathrm{cm}$ & $\begin{array}{c}\Lambda \\
\text { s.eq }^{-1} \cdot \mathrm{cm}^{2}\end{array}$ & $\mathrm{~K}\left(\min ^{-1}\right)$ \\
\hline 0 & 4.49 & 1.1225 & \multirow{16}{*}{$1.2 \times 10^{-3}$} \\
\hline 2 & 4.40 & 1.11 & \\
\hline 4 & 4.43 & 1.1075 & \\
\hline 6 & 4.42 & 1.105 & \\
\hline 8 & 4.41 & 1.1025 & \\
\hline 10 & 4.39 & 1.0925 & \\
\hline 12 & 4.37 & 1.0925 & \\
\hline 14 & 4.35 & 1.0875 & \\
\hline 16 & 4.33 & 1.085 & \\
\hline 18 & 4.33 & 1.0845 & \\
\hline 20 & 4.33 & 1.0838 & \\
\hline 22 & 4.33 & 1.0833 & \\
\hline 24 & 4.33 & 1.0825 & \\
\hline 26 & 4.34 & 1.0820 & \\
\hline 28 & 4.35 & 1.0812 & \\
\hline 30 & 4.35 & 1.0808 & \\
\hline
\end{tabular}

Table (4)

state conductivity values and rate constant for the effect of oxidation factors at [iodoforme] $=5 \times 10^{-4}$

\begin{tabular}{|c|c|c|c|}
\hline Time(min) & $\mathrm{L} \mu \mathrm{S} / \mathrm{cm}$ & $\begin{array}{c}\Lambda \\
\text { s.eq }^{-1} . \mathrm{cm}^{2}\end{array}$ & $\mathrm{~K}\left(\mathrm{~min}^{-1}\right)$ \\
\hline 0 & 30.4 & 0.608 & \multirow{16}{*}{$5 \times 10^{-5}$} \\
\hline 2 & 30.5 & 0.610 & \\
\hline 4 & 30.55 & 0.611 & \\
\hline 6 & 30.6 & 0.612 & \\
\hline 8 & 30.64 & 0.6128 & \\
\hline 10 & 30.7 & 0.614 & \\
\hline 12 & 30.77 & 0.6154 & \\
\hline 14 & 30.8 & 0.616 & \\
\hline 16 & 30.83 & 0.6166 & \\
\hline 18 & 30.9 & 0.618 & \\
\hline 20 & 30.97 & 0.6194 & \\
\hline 22 & 30.99 & 0.6198 & \\
\hline 24 & 31.03 & 0.6206 & \\
\hline 26 & 31.05 & 0.621 & \\
\hline 28 & 31.07 & 0.6214 & \\
\hline 30 & 31.12 & 0.622 & \\
\hline
\end{tabular}


Table (5)

state conductivity values and rate constant for the effect of oxidation factors at [iodoforme] $=6 \times 10^{-4}$

\begin{tabular}{|c|c|c|c|}
\hline Time(min) & $\mathrm{L} \mu \mathrm{S} / \mathrm{cm}$ & $\begin{array}{c}\Lambda \\
\text { s.eq }^{-1} \cdot \mathrm{cm}^{2}\end{array}$ & $\mathrm{~K}\left(\mathrm{~min}^{-1}\right)$ \\
\hline 0 & 3.74 & 0.62 & \multirow{16}{*}{$2.8 \times 10^{-4}$} \\
\hline 2 & 3.80 & 0.633 & \\
\hline 4 & 3.82 & 0.636 & \\
\hline 6 & 3.86 & 0.64 & \\
\hline 8 & 3.91 & 0.65 & \\
\hline 10 & 3.96 & 0.660 & \\
\hline 12 & 3.98 & 0.663 & \\
\hline 14 & 4.02 & 0.67 & \\
\hline 16 & 4.06 & 0.676 & \\
\hline 18 & 4.08 & 0.68 & \\
\hline 20 & 4.10 & 0.683 & \\
\hline 22 & 4.12 & 0.686 & \\
\hline 24 & 4.14 & 0.69 & \\
\hline 26 & 4.18 & 0.696 & \\
\hline 28 & 4.22 & 0.703 & \\
\hline 30 & 4.28 & 0.713 & \\
\hline
\end{tabular}

The data obtained also showed that the electron mobility between these two molecules, is responsible for that increase the table (1-4) show that molar conductance values, decrease with increasing of time at $\left(2 \times 10^{-4}-4 \times 10^{-4}\right)$ concentration, this refers to the conductivity of reaction, having a few conductivity, so the polarization of carbonyl group would also be decreased effecting by the inductive effect of the halogen. The attraction will stay keeping on randomly, the attraction and electrostatic forces would be having importance greatly and widely. The presence of an ionic atmosphere that combine the ions around the drug ketone and the opposite ions, the importance of ionic atmosphere, decreasing in case of increasing an infinite dilution of the solution until completely disappear, and this atmosphere effects ions mobility by increasing the concentration and due to presence unsymmetrical and electrophoretic effect which slow the mobility of ions, so it is noticed that molar conductance, decreased by increasing of time. while at $\left(5 \times 10^{-4}-6 \times 10^{-4}\right)$ the value of molar conductance would be increasing, due to the reaction in presence of the basic medium, effecting to the concentration of ketone, deading to increasing of the basicity of ketone, so the conductivity would be increasing the concentration on the formation of carboxylate ion as, stated in the previous mechanism, that is shown in the following figures. 


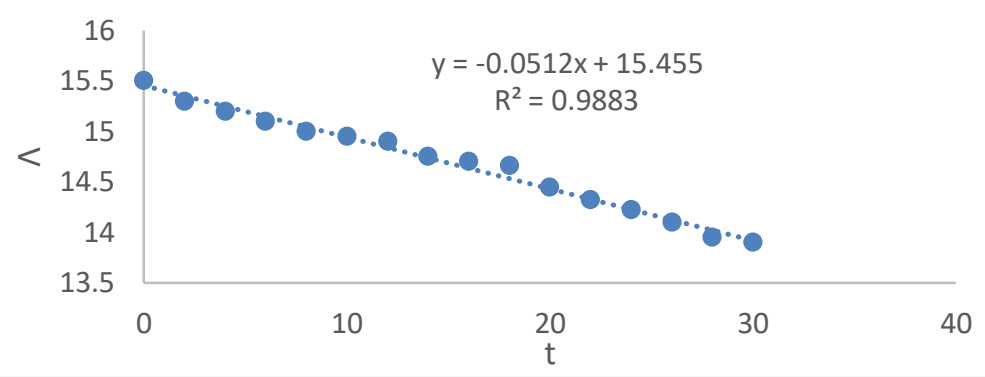

Fig(1) : effect of iodoform oxidation factor at $\left(2 \times 10^{-4}\right)$ molar con.

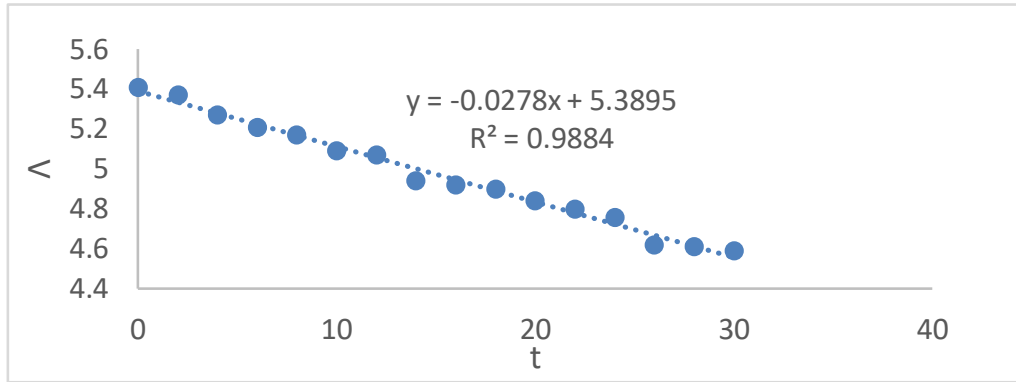

Fig(2) : effect of iodoform oxidation factor at $\left(3 \times 10^{-4}\right)$ molar con.

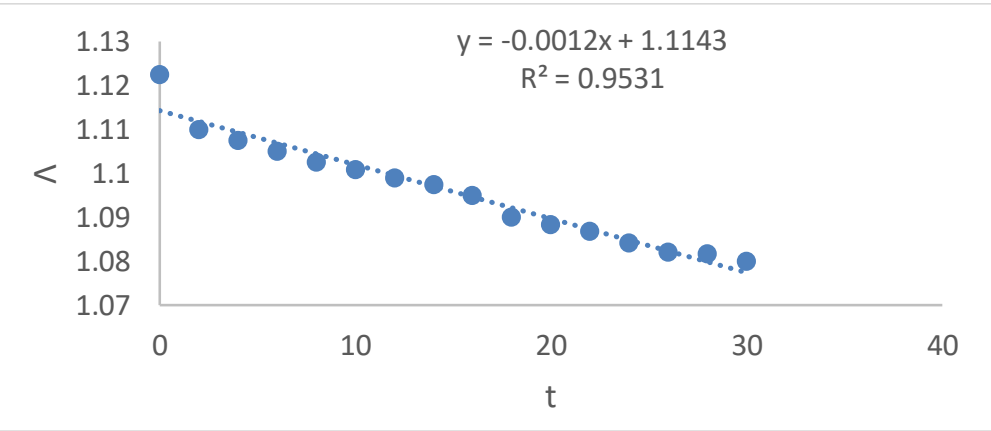

Fig(3) : effect of iodoform oxidation factor at $\left(4 \times 10^{-4}\right)$ molar con.

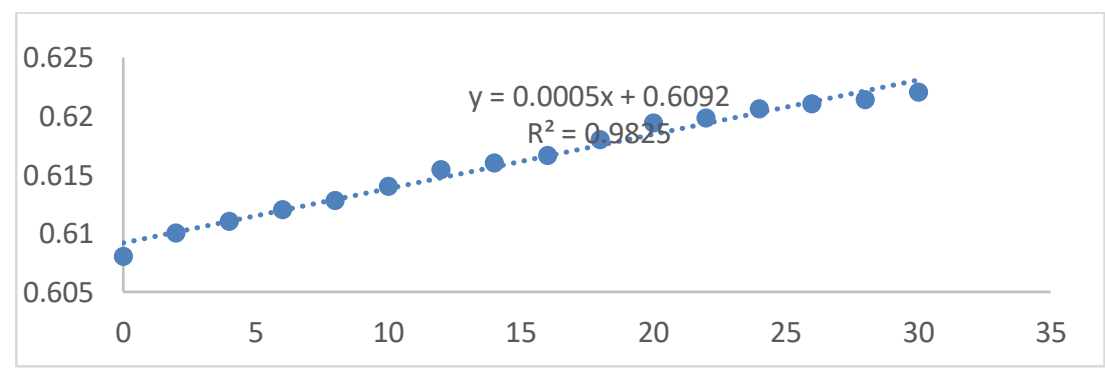

Fig(4) : effect of iodoform oxidation factor at $\left(5 \times 10^{-4}\right)$ molar con. 


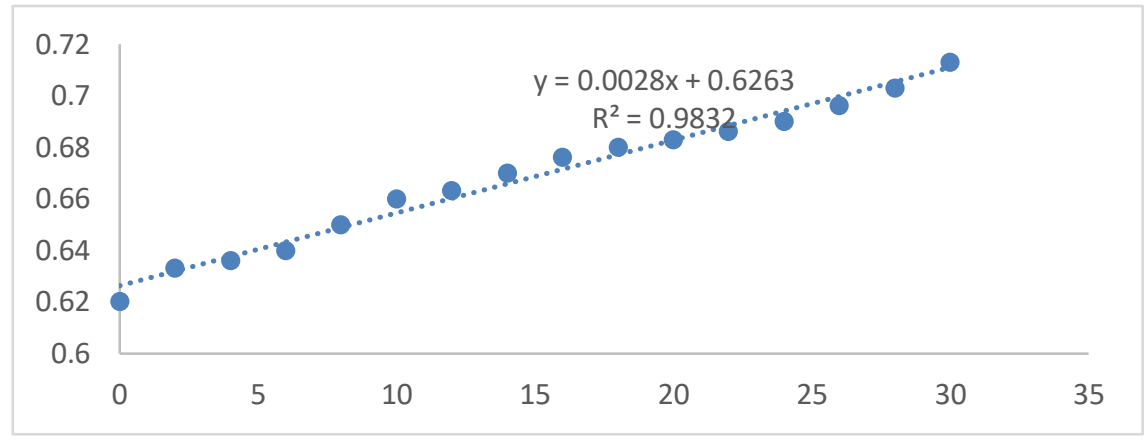

Fig(5): effect of iodoform oxidation factor at $\left(6 \times 10^{-4}\right)$ molar con.

Figure (6) show the stability constant (k) decreases with concentration increasing .because of the steric effect and the attraction and electrostatic forces.

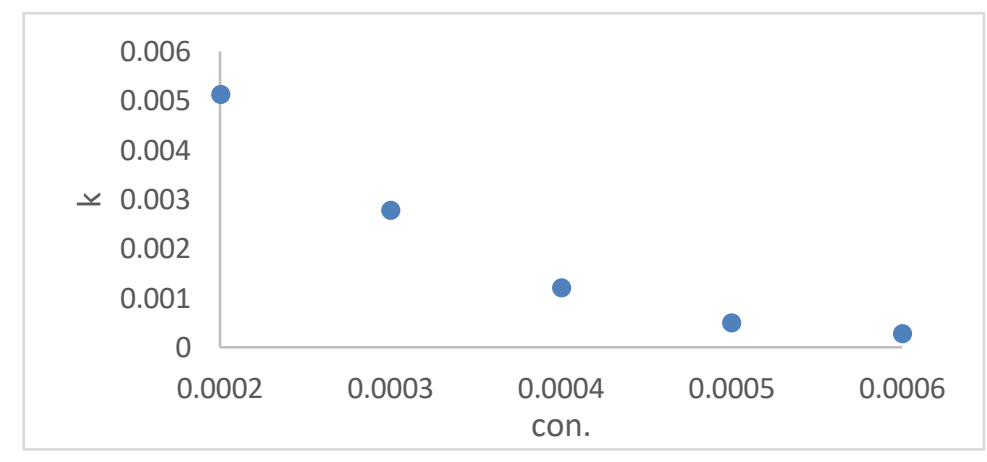

Fig $(6): k$ plotted against con.at oxidation process

Table (6)

Molar conductance values and molar values at infinite dilution and dissociation degree

\begin{tabular}{|c|c|c|c|c|c|c|}
\hline C & $\begin{array}{c}\mathrm{C}^{1 / 2} \\
\mathrm{X} 10^{-4}\end{array}$ & $\begin{array}{c}\text { Stability } \\
\text { time }\end{array}$ & $\Lambda$ & $\Lambda^{\circ}$ & $\boldsymbol{\alpha}$ & Kc \\
\hline $2^{x} 10^{-4}$ & 0.414 & 30 & 13.6 & \multirow{5}{*}{14.22} & 0.956 & $4.165 \times 10^{-3}$ \\
\hline $3^{\times} 10^{-4}$ & 0.732 & 30 & 4.59 & & 0.322 & $4.58 \times 10^{-5}$ \\
\hline $4 \times 10^{-4}$ & 2 & 30 & 1.0808 & & 0.0760 & $1.875 \times 10^{-6}$ \\
\hline $5^{\times} 10^{-4}$ & 2.23 & 30 & 0.622 & & 0.0437 & $9.984 \times 10^{-7}$ \\
\hline $6^{\mathrm{x}} 10^{-4}$ & 2.449 & 30 & 0.713 & & 0.0501 & $3.0180 \times 10^{-6}$ \\
\hline
\end{tabular}




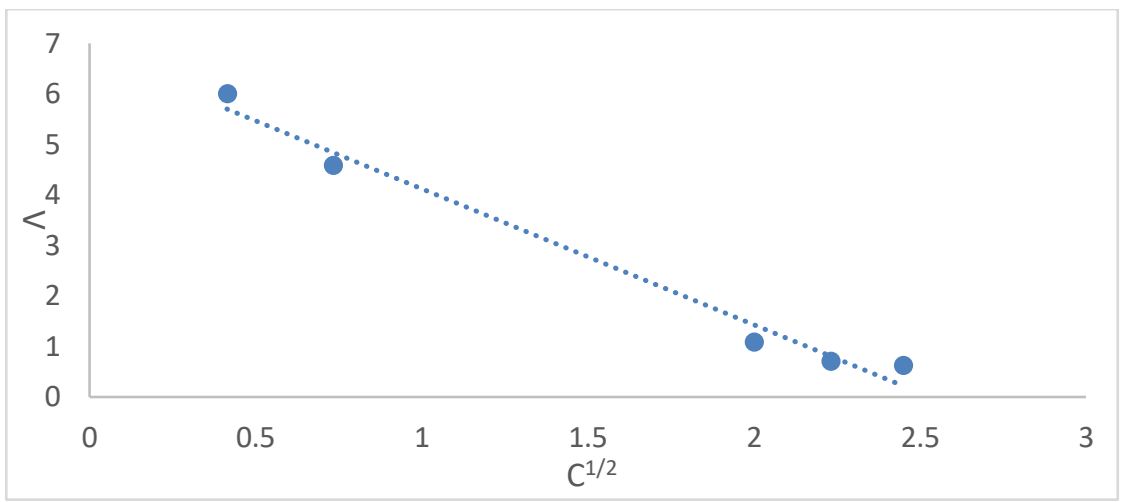

Fig(7) molar conductance values at infinite dilution via kohlrausch equation

Helmy's equation is :

$$
\ln \Lambda=\ln \Lambda_{0}-\left(\frac{\alpha+\beta}{\Lambda_{0}}\right) \sqrt{C}
$$

The equation is calculated $\Lambda_{0}$ value for the weak and very weak electroplate from Onsacers equation $(\Lambda-\sqrt{C})$, also the calculated values by which values were calculated more easily than the values calculated by the Kohlrausch'sequations since both had sometimes given a negative assessment of the equivalent or initial molar conductivity. Figure (8)

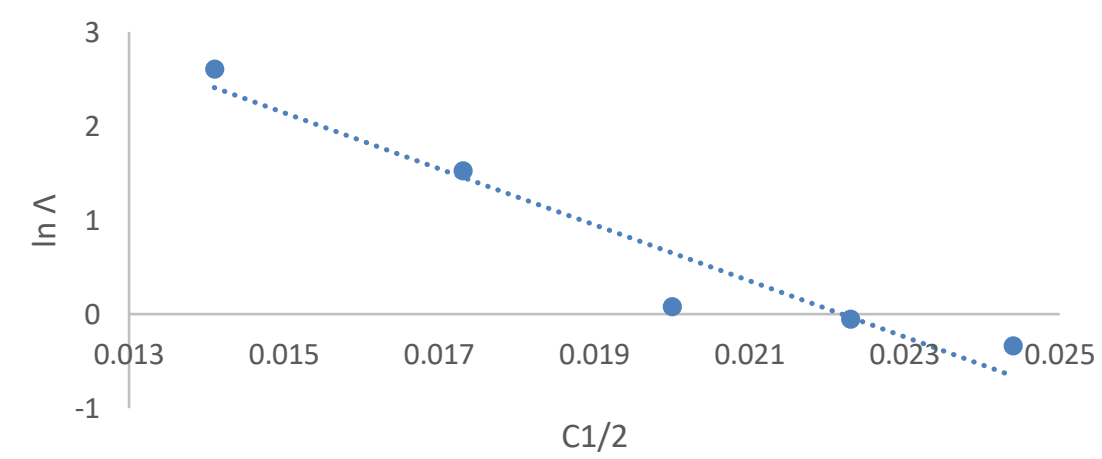

Fig (8) Helmy's theory of oxidation solutions were applied in ethanol for the initial infinity Molaric conductivity noat $25^{\circ}$.

\section{2- Results of Kineticexperiments}

The kinetic study had been performed at a basic medium between ketone drug and iodoform as shown in the following results: 
Table (7)

absorptivity values and reaction constant rate to oxidation factor at concentration $2 \times 10^{-4}$

\begin{tabular}{|c|c|c|c|}
\hline Time(min) & $\mathbf{A}$ & $\log \frac{A_{\circ}}{A_{t}}$ & $\mathbf{K}(\mathbf{m i n})$ \\
\hline 0 & 0.140 & 0 & \multirow{9}{*}{$1.5 \times 10^{-4}$} \\
\hline 5 & 0.145 & -0.01 & \\
\hline 10 & 0.147 & -0.02 & \\
\hline 15 & 0.149 & -0.027 & \\
\hline 20 & 0.151 & -0.032 & \\
\hline 25 & 0.154 & -0.04 & \\
\hline 30 & 0.155 & -0.045 & \\
\hline 35 & 0.159 & -0.055 & \\
\hline 40 & 0.163 & -0.065 & \\
\hline
\end{tabular}

Table (8)

absorptivity values and reaction constant rate to oxidation factor at concentration $3 \times 10^{-4}$

\begin{tabular}{|c|c|c|c|}
\hline Time(min) & $\mathbf{A}$ & $\log \frac{A_{\circ}}{A_{t}}$ & $\mathbf{K}(\mathbf{m i n})$ \\
\hline 0 & 0.032 & 0 & \multirow{16}{*}{$3.3 \times 10^{-5}$} \\
\hline 5 & 0.033 & -0.013 & \\
\hline 10 & 0.034 & -0.026 & \\
\hline 15 & 0.035 & -0.039 & \\
\hline 20 & 0.036 & -0.051 & \\
\hline 25 & 0.037 & -0.063 & \\
\hline 30 & 0.038 & -0.074 & \\
\hline 35 & 0.039 & -0.086 & \\
\hline 40 & 0.040 & -0.096 & \\
\hline 45 & 0.042 & -0.118 & \\
\hline 50 & 0.043 & -0.126 & \\
\hline 55 & 0.046 & -0.158 & \\
\hline 60 & 0.048 & -0.176 & \\
\hline 65 & 0.051 & -0.202 & \\
\hline 70 & 0.055 & -0.235 & \\
\hline 75 & 0.058 & -0.258 & \\
\hline
\end{tabular}


Table (9)

absorptivity values and reaction constant rate to oxidation factor at concentration $4 \times 10^{-4}$

\begin{tabular}{|c|c|c|c|}
\hline Time(min) & $\mathbf{A}$ & $\log \frac{A_{\circ}}{A_{t}}$ & $\mathbf{K}(\mathbf{m i n})$ \\
\hline 0 & 0.224 & 0 & \multirow{9}{*}{$1.0 \times 10^{-4}$} \\
\hline 5 & 0.228 & -0.007 & \\
\hline 10 & 0.231 & -0.012 & \\
\hline 15 & 0.232 & -0.015 & \\
\hline 20 & 0.234 & -0.018 & \\
\hline 25 & 0.237 & -0.024 & \\
\hline 30 & 0.242 & -0.033 & \\
\hline 35 & 0.243 & -0.035 & \\
\hline 40 & 0.246 & -0.040 & \\
\hline
\end{tabular}

Table (10)

absorptivity values and reaction constant rate to oxidation factor at concentration $5 \times 10^{-4}$

\begin{tabular}{|c|c|c|c|}
\hline Time(min) & $\mathbf{A}$ & $\log \frac{A_{\circ}}{A_{t}}$ & $\mathbf{K}(\min )$ \\
\hline 0 & 0.314 & 0 & \multirow{9}{*}{$3 \times 10^{-5}$} \\
\hline 5 & 0.315 & -0.0017 & \\
\hline 10 & 0.317 & -0.0043 & \\
\hline 15 & 0.318 & -0.0056 & \\
\hline 20 & 0.319 & -0.0070 & \\
\hline 25 & 0.320 & -0.0083 & \\
\hline 30 & 0.321 & -0.0096 & \\
\hline 35 & 0.322 & -0.010 & \\
\hline 40 & 0.323 & -0.021 & \\
\hline
\end{tabular}

Table (11)

absorptivity values and reaction constant rate to oxidation factor at concentration $6 \times 10^{-4}$

\begin{tabular}{|c|c|c|c|}
\hline Time(min) & $\mathbf{A}$ & $\log \frac{A_{\circ}}{A_{t}}$ & $\mathbf{K}(\mathbf{m i n})$ \\
\hline 0 & 0.177 & 0 & \multirow{9}{*}{$2.2 \times 10^{-4}$} \\
\hline 5 & 0.176 & 0.0024 & \\
\hline 10 & 0.172 & 0.0124 & \\
\hline 15 & 0.166 & 0.027 & \\
\hline 20 & 0.161 & 0.041 & \\
\hline 25 & 0.157 & 0.052 & \\
\hline 30 & 0.153 & 0.063 & \\
\hline 35 & 0.149 & 0.074 & \\
\hline 40 & 0.145 & 0.086 & \\
\hline
\end{tabular}




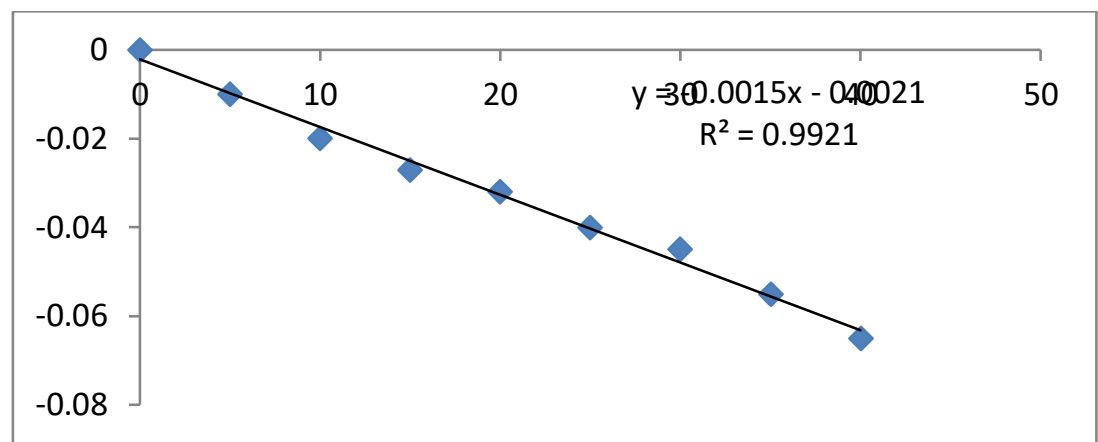

Fig (9) the relationship of the first-order reaction between drug ketone and iodoform at $2 \times 10^{-4}$

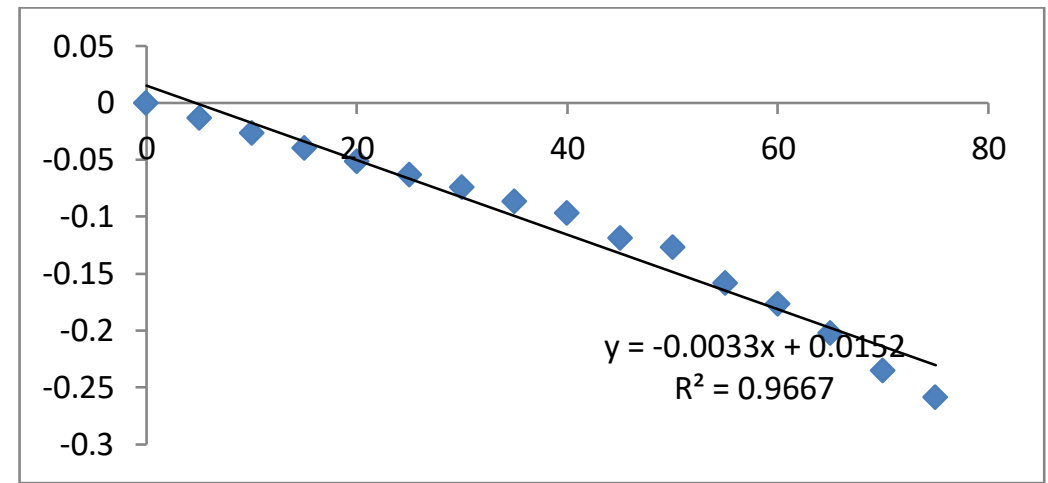

Fig (10) the relationship of the first-order reaction between drug ketone and iodoform at $3 \times 10^{-4}$

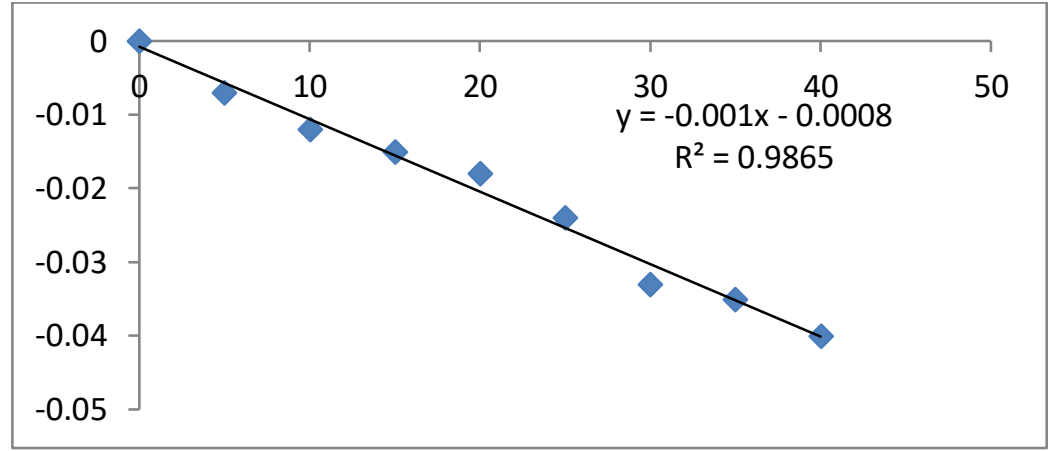

Fig (11) the relationship of the first-order reaction between drug ketone and iodoform at $4 \times 10^{-4}$

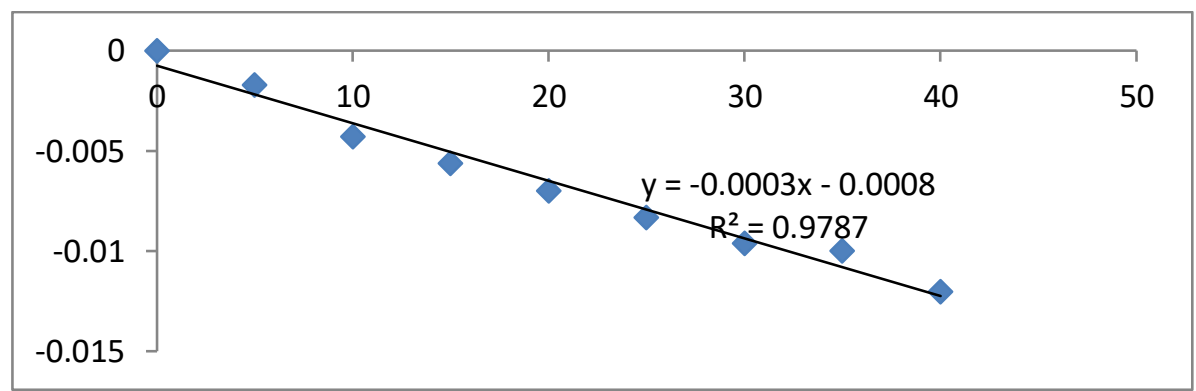

Fig (12) the relationship of the first-order reaction between drug ketone and iodoform at $5 \times 10^{-4}$ 
According to data obtained in tables (7-11) and figures $(9-12)$ it is clear that the mechanical reaction, taking place, is first order reaction during $\mathrm{k}$ values obtained from the following equation $\mathrm{k}=\frac{2.303}{\mathrm{t}} \log \frac{A_{\circ}}{A_{t}}$ and clear correlation factor in all figures, having the values $m$ ranged (0.9921-0.9667), wave lengths of the prepared solution, were measured according to the wavelength of drug ketone and iodoform oxidized factor and also the kind of used solvent , the peaks wave (325nm) and(363nm) respectively, this change to new peak at $498 \mathrm{~nm}$ as indication to obtain the electronic transition in this reaction as $\left(n-\pi^{*}\right)$, which is transferring the electrons between the drug ketone and oxidized factor in basic medium [16-19]. According to mechanism of reaction, it is showed that presence of cage effect and solvation effect which are effecting to the absorption peaks directly. These factors basically depend on nature of material and solvent used to solve the material, so this leads to combine solvent molecules round the reacted material which can be explained as surrounding for obstical the molecules to interact with each other, which can be noticed in case of decreasing the absorptivity, changing charge for dissimilar of ions that can positively and negatively effecting to the stability of formed compounds[20] .

In conclusion, we can say that the solvent has a great effect $m$ which can explain the contrast in the values of absorption, exclusively in polar solvents as aversely to the ability of the compound to interact with polar solvents because they have functional groups like carbonyl one and that is because of cage effect.

Previous studies have the In the present investigation we have studied the kinetics and mechanism of oxidation of ester by potassium dichromate in acid medium. In this study, we reported the effect of oxidant $\mathrm{K}_{2} \mathrm{Cr}_{2} \mathrm{O}_{7}$, the effect of substrate (ester), the effect of sulphuric acid, and the effect of temperature on the oxidation of ester. The reaction was first order according to oxidant and substrate, as temperature increases rate of the reaction also increases. and second research shows the Permagnetic oxidation of 3Ethoxy-4- Hydroxybenzaldehyde has been studied at different temperatures using spectrophotometer under acidic conditions. The effect of variation of a substrate (3-E-4-HB), oxidant $\left(\mathrm{KMnO}_{4}\right)$ andH $\mathrm{SO}_{4}$ was studied under pseudo-first-order reaction conditions. The effect of different salts on the oxidation of 3-E-4-HB was also studied. The reaction was found to be first order to oxidant, substrate, and H2SO4. A suitable mechanism is also suggested for the oxidation reaction.

\section{References}

1- Tarakkaheaa, "Chemical and industrial processes" p.21, 1999.

2- Basheer KM, Joseph J, Radhakrishnan Nair TD, Structure- reactivity correlation and mechanistic aspects of the $\mathrm{Cr}(\mathrm{VI})$ oxidation of benzhydrols, Int. J. Chem. Sci., 5(3), 2007, 1191-1197

3- Omar A.H.," electrochemistry " part (1), pp:1618, 2013.

4- Guido Kickelbick: ChemiefürIngenieure. 1. Auflage. Pearson Studium, p. 176, 2008 .

5- Bijudas K, Kinetics and mechanism of the selective oxidation of benzyl alcohols by acidified dichromate in aqueous acetic acid medium, Oriental J. Chem., 30 (3), 2014, 13911396. DOI: $10.13005 /$ jc/300360

6- A.B.T.kreshoov, A.yaroSlaftsev, Translation: EsaaMasoh, "analysis chemistry" Dar Al Amir Printing and Publishing, pp:170-172, 1982.

7- James, Frank A. J. L. "Cooper, John Thomas (1790-1854), chemist". Oxford Dictionary of National Biography. Oxford University Press. Retrieved 26 January 2012.

8- V. S. BAGOTSKY, Fundamentals of Electrochemistry, Second Edition, A. N. Frumkin Institute of Physical Chemistry and Electrochemistry, Russian Academy of Sciences, Moscow, Russia

9- SayyedHussin, B.R.Agrawal, S.B. Pakhare, MazherFarooqi," kinetic and mechanistic study of oxidation of ester by $\mathrm{K}_{2} \mathrm{Cr}_{2} \mathrm{O}_{7}$ ", Int. J. Chem. Res., vol 2, Issue 2, (2011).

10- Chekanov, Y; Ohanogi, R, Asai, S. Polym., J., 30 (5), 3811 (1998)

11- Von Gunten, Oxidation kinetics, and product formation, Elsevier, Volume 37, Issue 7, April 2003, Pages 1443-1467.

12- Siddharth B. Pakhara, MilindUbale, Jaishree Gawaiand MazaharFarooqi, "kinetic and mechanistic oxidation of 3-ethoxy-4hydroxybenzaldehyde using KMnO4" rasayanJ.chem., vol 8,no1,p123-132, (2015).

13- Najwa Abdul Aziz Saeed Awn, Maqdoom Farooqi, S.B. Pakhare, and Mazhar Farooqi, "kinetic of permagneric pyridine -2carboxaldehyde in acidic media " J. of Med.chem.and drug discovery, ISSN: 23479027, (2015).

14- T.Dziembowska, E. Jagodazinska, Z.Rozwadowski and M.Kotfica "Solvent Effect On Intramolecular Proton Transfer equilibrium in some N.(R-Salicylidene)-alkyl amine ", J.Mol.Struct,598,229. (2001). 
15- SairabanuA.FarokhiSharanappaT.

Nandibewoor , Kinetic, mechanistic and spectral studies for the oxidation of sulfanilic acid by alkaline hexacyanoferrate(III), Tetrahadron, J., Volume 59, Issue 38, 15 September 2003, Pages 7595-7602

16- L.Antonov, W.M.Fabian, D.Nedeltcheva, and F.S.Kamounah, "Tautomersimof 2-hydroxyl naphthaldehyde Schiff bases"J.Chem.Soc,2,1173-1179,(2000).

17- H.J.salvagione, J.Arias, P.Garces "spectroelectrochemical study of the oxidation of aminophenols on platinum electrode in acid medium" ,J.Electroanalyticalchem.vol(565), issue 2,p375-383,2004 .

18- Sayyed. H, MazharF, Gaikwad D, " kinetic and mechanistic study of oxidation of ester by $\mathrm{KMnO}_{4}$ " int.J.of chem. Res. ,vol2, no1,pp242$249,(2010)$.

19- Sayyed .H, MazharF,Gaikwad D, "kinetic and mechanistic study of oxidation of 2,5-giamino1,3,4-thiadazole frrate complex in acid medium" ,vol(3),no(1),p13-19, 2012 .

20- YousifAlgamal, M.A.Embaby, Majed H. Shtaiwi, "Cooxidation of Chalcones and Oxalic acid using Chromium (VI)", International Journal of Sciences, ISSN 2307-4531, (2014) Volume 15, No 1, pp 66-79.

21- M. SudhaSwaraga, M. VasudevanShruthiandM. SnehaBhavani, Kinetic and mechanistic study of oxidation of purines by sodium chromate and hydrogen peroxide system in presence of ascorbic acid and protection by riboflavin, Int. ISSN 0972-768X, J. Chem. Sci.: 14(4), pp: 2420-2432, 2016. 